\title{
Major Depression and Allergy: Does Neuroticism Explain the Relationship?
}

\author{
Renee D. Goodwin, PhD, MPH, Mario Castro, MD, MPH, and Monika Kovacs, MD
}

\begin{abstract}
Objective: To determine the association between major depression, neuroticism, and self-reported allergy among adults in the community. Methods: Data were drawn from the Midlife Development in the United States Survey, a nationally representative sample of 3,032 adults age 25 to 74 . ANOVA and multiple logistic regression analyses were used to determine the association between depression and allergy and the role of neuroticism in these relationships. These links were also examined by gender. Results: Among adults in the community, major depression was associated with a significantly increased likelihood of allergy (odds ratio $[\mathrm{OR}], 1.5 ; 95 \%$ confidence interval [CI], 1.14-1.98). Higher levels of neuroticism were also significantly associated with increased likelihood of allergy (OR, 1.22; 95\% CI, 1.04-1.43). Among women, major depression was associated with a significantly increased likelihood of allergy (OR, 1.67; 95\% CI, 1.15-2.41), and this relationship persisted after adjusting for demographic characteristics and neuroticism. Among men, there was no significant relationship between allergy and depression, yet neuroticism was related to allergy (OR, 1.42; 95\% CI, 1.42-1.82), which persisted after adjustment for depression. Conclusion: These data are consistent with results of previous studies showing an association between major depression and allergy among adults and extend these data by providing preliminary evidence suggesting that this association is specific to women and independent of the effects of neuroticism among women. In addition, the data provide preliminary evidence that neuroticism may be related to allergy among men, though no link between depression and allergy was found among men. Future research with prospective, longitudinal studies is needed next to understand the possible biological underpinnings of these associations. Key words: epidemiology, major depression, allergy, comorbidity, neuroticism, mental disorders, personality factors.
\end{abstract}

MIDUS = Midlife Development in the United States; CIDI = Composite International Diagnostic Interview; CIDI-SF = Composite International Diagnostic Interview Short Form.

\section{INTRODUCTION}

P revious studies have shown an association between atopic disease and depression (1-6). These data come mainly from four sources. First, data from clinical studies have shown that allergy patients show higher levels of depressive symptoms (5) compared with control groups. For instance, Kovacs et al. (6) found higher levels of depressive symptoms among allergy patients compared with those without allergy. Second, data from psychiatric clinic patients have reported a higherthan-expected level of allergic disease among patients with depression. Third, data from twin studies suggest a genetic link between atopy and depression. Wamboldt et al. (2) reported a familial link between atopy and depression in Finnish adults twins. Finally, several epidemiologic studies have shown links between allergy and depression in nonclinical samples $(1,3,4)$. Timonen et al. (3) recently provided strong evidence of an association between allergy and depression in a community sample, using methodology that included allergy tested with skin-prick test and clinically diagnosed depression in a birth cohort study. Moreover, a number of studies have found significant gender differences in the relationship between allergy and depression among females. The link between allergy and depression was not evident among males except in the presence of more severe symptoms of depression $(3,7)$. This finding of a link between allergy and depression in females has been replicated $(3,7)$. In addition, associations

From the Department of Epidemiology, Mailman School of Public Health, Columbia University, New York, NY (R.D.G.); Department of Pulmonology and Critical Care Medicine, Washington University, St. Louis, MO (M.C.); and Semmelweis Institute, Budapest, Hungary (M.K.).

Address correspondence and reprint requests to Renee D. Goodwin, PhD, MPH, Department of Epidemiology, Mailman School of Public Health, Columbia University, 1051 Riverside Dr., Unit 43, New York, NY 10032. E-mail: rdg66@columbia.edu

Received for publication July 22, 2004; revision received July 22, 2005.

DOI: 10.1097/01.psy.0000195797.78162.f4 have been found between allergy and increased rates of depression and anxiety among adults in clinical samples $(6,11,12)$.

Similarly, other data have suggested a link between personality and temperament and the presence of allergic diseases. Several reports by Keogh (8), Bell et al. (9), Kagan et al. (10), and Gauce et al. (11) have found an association between self-reported allergies and increased likelihood of behavioral inhibition among youth. In one study, preschool children with a history of atopy had significantly higher levels of behavioral inhibition compared with those without atopy (9). A familial association between atopy and behavioral inhibition has also been documented (10). Associations between specific personality factors and risk of mental disorders have been noted in several population-based studies $(13,14)$. For instance, neuroticism is associated with increased risk of psychotic disorders (13) and with anxiety and depressive disorders $(14,15)$.

Personality factors are thought to develop early in life and to play a fundamental role in patterns of behavior and emotional functioning, which are relatively consistent throughout the lifespan. Some personality factors are thought to be linked with both physical and mental disorders (e.g., neuroticism). Therefore, it is conceivable that the co-occurrence of mental and physical disorders (e.g., allergy and depression) could be related to result from the presence of specific personality factors (i.e., neuroticism) that increase the risk for both. Previous studies, however, have not examined the influence of neuroticism on the co-occurrence of allergy and depression. Finally, although previous studies have shown links between depressive symptoms and participant report of physicians' diagnosis of major depression, previous studies have not included a standardized measure of depression using DSM criteria. Therefore, although previous studies have suggested a linear relationship between depression symptom severity and likelihood of allergy, the relationship between allergy and clinical depression (i.e., diagnosis based on DSM criteria) in a general population sample has not been thoroughly examined. 
Accordingly, the goal of the current study is to investigate the relationship between depression and allergy and the role of neuroticism in this association in a large representative sample in the United States. In addition, we investigated each of these relationships by gender. The rationale for examining gender differences was two-fold. One, previous studies have documented gender disparities in the link between allergy and depression among both adults $(3,7)$ and youth (9). In addition to these previous findings, the strong role of gender in depression suggested that gender may also be involved in these links.

The goal of this study was to begin to fill this gap by addressing four main questions. First, is there an association between self-reported allergy and major depression among adults in the community? Second, is there an association between allergy and neuroticism among adults in the community? Third, does the relationship between allergy, major depression, and neuroticism differ by gender? Fourth, does neuroticism explain the relationship between allergy and major depression? We hypothesized that there would be a relationship between allergy and major depression. Based on previous findings, we expected that this association may be more prominent among females than males.

\section{METHODS}

\section{Study Design}

The Midlife Development in the United States (MIDUS) Survey is a nationally representative survey of 3,032 persons age 25 to 74 years in the noninstitutionalized civilian population of the 48 coterminous United States $(16,17)$. The MIDUS Survey was carried out by the John D. and Catherine T. MacArthur Foundation Network on Successful Midlife Development between January 1995 and January 1996. All respondents completed a 30-minute telephone interview (70.0\% response rate) and filled out two mailed questionnaires estimated to take a total of about 90 minutes to complete $(86.8 \%$ conditional response rate in the subsample of telephone respondents). The overall response rate was $60.8 \%$. The data reported in this paper were weighted to adjust for differential probabilities of selection and nonresponse. More details on the MIDUS Survey design, field procedures, and sampling weights are available elsewhere $(16,17)$.

\section{Demographic Characteristics}

Self-reported data were gathered on age, gender, race, marital status, and level of educational attainment. For the purposes of these analyses, age was the only continuous variable (ranging from 25 to 74). Race (Caucasian and minority racial status); Marital status (married and unmarried); and educational attainment (high school diploma and did not complete high school) were collapsed into dichotomous variables.

\section{Diagnostic Assessment}

The MIDUS Survey diagnoses were based on the Composite International Diagnostic Interview Short Form (CIDI-SF), a series of diagnostic-specific scales that were developed from item-level analyses of the Composite International Diagnostic Interview (CIDI) questions in the National Comorbidity Survey (NCS) (18-20). The CIDI-SF was designed to reproduce the full CIDI diagnoses as well as possible with only a small subset of the original questions. Details of specificity and sensitivity of CIDI-SF classifications are available elsewhere (20). CIDI-SF was used to diagnose major depression (past 12-months). Participants were presented with a list of physical illnesses, which included hay fever (allergy), as well as asthma/chronic bronchitis, tuberculosis, other lung disease, hypertension, AIDS, diabetes, hernia, stomach problems, ulcer, autoimmune disorders, neurological disorders, persistent problems with teeth and gums, and migraine headaches and were asked to endorse any or all of those which they had experienced or been diagnosed with in the past 12 months.

\section{Personality Factors}

Assessment of personality traits in the MIDI, based on the "big five" factor model, was developed using the results of a pilot study, which was conducted in 1994 with a probability sample of 1,000 men and women, age 30 to 70 (574 valid cases were usable for item analysis) (21-24). Items with the highest item to total correlations and factor loadings were selected for MIDI. Forward regressions were also conducted to determine the smallest number of items needed to account for over $90 \%$ of the total scale variance. Scales included agreeableness (helpful, warm, caring, softhearted, sympathetic) $(\alpha=0.80)$ (5-item scale); openness to experience (creative, imaginative, intelligent, intelligent, curious, sophisticated, adventurous) ( $\alpha=0.77$; 7-item scale); conscientiousness (organized, responsible, hardworking, [not] careless) ( $\alpha=0.57$; 4-item scale); extraversion (outgoing, friendly, lively, active, talkative) ( $\alpha=0.78 ; 5$-item scale); and neuroticism (moody, worrying, nervous, [not] calm) $(\alpha=0.74 ; 4$-item scale $)$. The $\alpha$ s are based on the MIDUS national sample.

\section{Analytic Strategy}

First, the association between allergy and major depression was examined using F-based tests of independence, with significance set at 0.05 . We examined the differences in mean (SD) of neuroticism between those with and without allergy using ANOVA. Next, multiple logistic regression analyses were used to determine the association between allergy and depression. First, unadjusted analyses were run to calculate odds ratios (with $95 \%$ confidence intervals) of the link between allergy and depression. Next, these analyses were adjusted for demographic differences (i.e., gender, age, race, martial status, education); the analyses were then further adjusted for neuroticism. The same analyses were then rerun with the sample stratified by gender.

\section{RESULTS}

\section{Prevalence and Sociodemographic Characteristics}

Six hundred respondents (14\%) reported having allergy and $617(14.2 \%)$ reported depression. Those with allergy were younger (45.6 (12.2) versus $47.2(13.2)$ years old, $p=.03)$ than those without allergy. There were no significant differences in gender, race, education, or marital status between adults with and without allergy.

\section{Association Between Major Depression, Neuroticism, and Allergy Among Adults}

Major depression was associated with a significantly increased likelihood of allergy, compared with those without allergy (18.9\% (79/418) versus $13.4 \%(296 / 2214) ; \chi^{2}=8.8$, $d f=1, p=.003)$. Allergy was also associated with significantly higher levels of neuroticism (M (SD), 2.33 (0.67) versus $2.24(0.66), \mathrm{F}=6.09, p=.014)$. After adjusting for demographics and neuroticism, the relationship between major depression and allergy persisted (OR, 1.36; $(1.02,1.81))$ (see Table 1). Similarly, the link between neuroticism and allergy remained after adjusting for demographics and major depression (OR, 1.18; $(1.0,1.39))$, suggesting that major depression and neuroticism are each independently related to allergy.

\section{Association Between Major Depression, Neuroticism, and Allergy Among Male Adults}

Among males, depression was not associated with allergy $\left(13.6 \%(27 / 196)\right.$ versus $10.5 \%(113 / 1067), \chi^{2}=1.7, d f=1$, 
TABLE 1. Association Between Major Depression, Neuroticism, and Allergy Among Adults in the Community

\begin{tabular}{lccc}
\hline & $\begin{array}{c}\text { Allergy, Unadjusted OR } \\
(95 \% \mathrm{Cl})\end{array}$ & $\begin{array}{c}\text { Allergy, AOR1 } \\
(95 \% \mathrm{Cl})\end{array}$ & $\begin{array}{c}\text { Allergy, AOR2 } \\
(95 \% \mathrm{Cl})\end{array}$ \\
\hline Major depression & $1.5(1.14-1.98)^{*}$ & $1.5(1.13-1.98)^{*}$ & $1.36(1.02-1.81)^{*}$ \\
Neuroticism & $1.22(1.04-1.43)^{*}$ & $1.23(1.05-1.45)^{*}$ & $1.18(1.0-1.39)^{*}$ \\
\hline
\end{tabular}

$* p<.05$.

AOR1 = adjusted for differences in gender, age, race, marital status, and educational attainment. AOR2 = adjusted for demographic differences, depression, and neuroticism simultaneously.

$p=.19)$; but higher neuroticism was associated with a significantly increased likelihood of allergy (M (SD) 2.27 (0.64) versus $2.13(0.62), \mathrm{F}=8.7, p=.003))$. After adjusting for demographics, depression, and neuroticism, neuroticism remained significantly associated with increased likelihood of allergy. The link between depression and allergy was not significant among males (see Table 2). After adjusting for demographics, the link between neuroticism and allergy remained statistically significant and persisted after adjusting for depression (OR, $1.42(1.1,1.82))$.

\section{Association Between Major Depression, Neuroticism, and Allergy Among Female Adults}

Major depression was associated with a significantly increased likelihood of allergy among females (23.6\% (52/220) versus $\left.16.1 \%(183 / 1129), \chi^{2}=7.3, d f=1, p=.007\right)$. Neuroticism was not directly linked with allergy among females (M (SD) 2.38 (0.69) versus 2.35 (0.67), $\mathrm{F}=0.4, p=$ .5). After adjusting for demographics, the link between depression and allergy persisted (OR, 1.67; (1.14, 2.37)) and continued to remain significant after adjusting for neuroticism (OR, 1.63; (1.15, 2.41)) (see Table 3). The link between neuroticism and allergy was not statistically significant.

\section{DISCUSSION}

Consistent with previous results, these data suggest that there is a significant relationship between allergy and depression among females but not males in the community. This relationship persists after further adjustment for differences in demographic characteristics and appears independent of the effects of neuroticism. Our results also provide preliminary evidence that there is a link between neuroticism and increased likelihood of allergy among adults and that this association appears specific to males and independent of the effects of major depression. These links have not previously been demonstrated among a community-based population of adult in the United States.

The mechanism of association between allergy and depression in females cannot be determined due to the crosssectional nature of these data. Although these findings are consistent with those of Timonen $(3,7)$, they add to existing knowledge by replicating this gender difference in a sample of adults into older adulthood, whereas previous studies have been limited to relatively early adulthood. Therefore, the data suggest that age does not appear to play a role in this link and that it is consistent across age groups. These data also contribute to existing knowledge on the link between allergy and depression by providing data on depression collected using a well-validated epidemiologic instrument to assess depression using DSM criteria $(20,25)$. Previous studies have relied on symptom measures and participant recall of having been given a diagnosis of depression by a physician during their lifetime, which are subject to treatment-seeking and recall biases. These results describe the relationship between allergy and past 12 months of depression, according to a standardized measure using DSM criteria $(20,25)$. The results do not support the hypothesis that neuroticism explains the link between depression and allergy but instead suggest the link between depression and allergy is independent of neuroticism in females and that neuroticism is directly related to allergy among males independent of depression.

These data provide preliminary evidence that neuroticism may play a role in the vulnerability to allergy among males in the community. The mechanism of this link, however, is not known. It may be that individuals with high levels of neuroticism are more likely to develop both allergy and depression. Numerous studies have shown linkages between neuroticism and increased depression (13-15), though less is known about the linkage between neuroticism and allergy. Yet, this possible relation is consistent theoretically with results from clinical

TABLE 2. Association Between Major Depression, Neuroticism, and Allergy Among Male Adults in the Community

\begin{tabular}{|c|c|c|c|}
\hline & $\begin{array}{l}\text { Allergy, Unadjusted OR } \\
\qquad(95 \% \mathrm{Cl})\end{array}$ & $\begin{array}{l}\text { Allergy, AOR1 } \\
\quad(95 \% \mathrm{Cl})\end{array}$ & $\begin{array}{c}\text { Allergy, AOR2 } \\
(95 \% \mathrm{Cl})\end{array}$ \\
\hline Major depression & $1.34(0.85-2.1)$ & $1.25(0.79-1.98)$ & $0.98(0.6-1.6)$ \\
\hline Neuroticism & $1.43(1.13-1.82)^{*}$ & $1.41(1.11-1.8)^{\star}$ & $1.42(1.1-1.82)^{*}$ \\
\hline
\end{tabular}

$* p<.05$.

AOR1 = adjusted for differences in age, race, marital status, and educational attainment; AOR2 = adjusted for demographic differences, depression, and neuroticism simultaneously. 
TABLE 3. Association Between Major Depression, Neuroticism, and Allergy Among Female Adults in the Community

\begin{tabular}{lccc}
\hline & $\begin{array}{c}\text { Allergy, Unadjusted OR } \\
(95 \% \mathrm{Cl})\end{array}$ & $\begin{array}{c}\text { Allergy, AOR1 } \\
(95 \% \mathrm{Cl})\end{array}$ & $\begin{array}{c}\text { Allergy, AOR2 } \\
(95 \% \mathrm{Cl})\end{array}$ \\
\hline Major depression & $1.61(1.14-2.28)^{*}$ & $1.67(1.15-2.41)^{*}$ & $1.63(1.14-2.32)^{*}$ \\
Neuroticism & $1.07(0.87-1.33)$ & $1.05(0.84-1.31)$ & $0.97(0.77-1.22)$ \\
\hline
\end{tabular}

$* p<.05$.

AOR1 = adjusted for differences in age, race, marital status, and educational attainment; AOR2 = adjusted for demographic differences, depression, and neuroticism simultaneously.

studies showing that behavioral inhibition is associated with increased allergy among male youth (9) and showing a familial association between behavioral inhibition and allergy (10). It may be that there is a common genetic vulnerability to both allergy and behavioral inhibition or neuroticism among males. Neuroticism and behavioral inhibition have been thought to be similar if not overlapping constructs. Alternatively, it may be that having high levels of neuroticism increases the likelihood of perceiving allergic responses to exposures that individuals with lower levels of neuroticism may not report. It is not known whether report bias or somatization occurs, (26), yet as high neuroticism is thought to be associated with perceiving the world and events in a negative light, those with high neuroticism may perceive symptoms more readily than those less sensitive with low neuroticism to such exposures or symptoms.

Another potential pathway that could explain this association is that allergy leads to functional impairment and distress in coping with uncomfortable and painful allergy symptoms daily, such that this leads to increased risk of depression onset. As allergy is chronic in the majority of cases (27), persisting for years, and has been shown to be associated with high levels of occupational disability and impairment in social relationships $(28,29)$, it is conceivable that allergy may contribute to the risk of depression. Similarly, although neuroticism as a personality trait is thought to develop early in life, it may be that having allergy leads to an increase in negative thoughts and response patterns to external stimuli due to daily stress and discomfort associated with having allergy. Alternatively, it is possible that depression increases vulnerability to developing allergy through weakening the immune system or another biological pathway, as has been suggested in various clinically based studies $(30,31)$. Still, none of these pathways fully address why these gender differences would emerge. The gender differences suggest that multiple pathways or a combination of pathways is more likely contributing or that there are other social, biological, or genetic factors at work.

Our findings are consistent with previous results showing consistent gender differences in the relationships between allergy and major depression in adults. As the data presented in this study are also cross-sectional and observational, rather than experimental, it is not possible to obtain evidence addressing the possibility of a causal relationship. Previous studies have shown strong differences in gender and depression and speculated extensively on the possible immunerelated pathways of this association (see Timonen et al. (3) and Kovacs et al. (6) for review). Although depression and allergy are strongly related in females, neuroticism does not appear to play a role in this link, nor is it directly related to allergy. In addition, the relationship between allergy and neuroticism among males persists, whereas there is no significant link between allergy and depression, and depression does not appear to affect the relationship between allergy and neuroticism. As such, these data may provide preliminary evidence suggesting that allergy is more strongly related to neuroticism in males and more strongly related to depression in females. These findings clearly require replication with more refined measures.

Limitations of this study are numerous and should be considered when interpreting these results. First, data on allergy was self-reported and therefore may be biased by tendencies toward overreporting among persons with depression, as previous studies suggest higher levels of somatic symptoms reported among persons with mental disorders compared with those without. It should be noted that depression was measured only for the past 12 months, rather than lifetime. Therefore, these limitations on the assessment of either or both depression and allergy could influence results. Second, information was available only on adults age 25 to 74 ; therefore these findings are not generalizable to other age groups. Future studies that can examine the linkages between personality traits, allergy, and depression using prospective, longitudinal epidemiologic data beginning in childhood with biological measures of allergy status and possibly on immune functioning, as well as longitudinal data on personality factors, major depression, and common behavioral risk factors for both, are needed next for replication of these findings and to further our understanding of the mechanism of these linkages, especially in uncovering the reason for gender differences in these relationships.

The authors would like to thank Jerome Kagan, PhD, for helpful comments on an earlier version of this manuscript.

\section{REFERENCES}

1. Bell IR, Jasnoski ML, Kagan J, King DS. Depression and allergies: survey of a nonclinical population. Psychother Psychosom 1991;55: 24-31.

2. Wamboldt MZ, Hewitt FK, Schmitz S, Wamboldt FS, Rasanen M, Koskenvuo M, Romanov K, Varjonen J, Kaprio J. Familial association between allergic disorders and depression in adult Finnish twins. Am J Med Genet 2000;96:146-53.

3. Timonen M, Jokelainen J, Silvennoinen-Kassinen S, Herva A, Zitting P, $\mathrm{Xu}$ B, Petola O, Rasanen P. Association between skin test diagnosed 
atopy and professionally diagnosed depression: a Northern Finland 1966 birth cohort study. Biol Psychiatry 2002;52:349-55.

4. Hurwitz EL, Morgenstern H. Cross-sectional associations of asthma, hay-fever, and other allergies with major depression and low back pain among adults aged 20-39 years in the United States. Am J Epidemiol 1999;150:1107-16.

5. Brown ES, Khan DA, Mahadi S. Psychiatric diagnosis in inner city outpatients with moderate-severe asthma. Int J Psychol Med 2000;30: 319-27.

6. Kovacs M, Stauder A, Szedmak S. Severity of allergic complaints: the importance of depressed mood. J Psychosom Res 2003;54:549-57.

7. Timonen M, Jokelainen J, Hakko H, Silvennoinen-Kassinen S, MeyerRochow VB, Herva A, Rasanen P. Atopy and depression: results from the Northern Finland 1966 Birth Cohort Study. Mol Psychiatry 2003;8: $738-44$.

8. Keogh B. Global strategy for asthma. Ir Med J 1998;91:76-7.

9. Bell IR, Jasnoski ML, Kagan J, King DS. Is allergic rhinitis more frequent in young adults with extreme shyness? a preliminary survey. Psychosom Med 1990;52:517-25.

10. Kagan J, Snidman N, Julia-Sellers M, Johnson MO. Temperament and allergic symptoms. Psychosom Med 1991;53:332-40.

11. Gauci M, King MG, Saxarra H, Tulloch BJ, Husband AJ. A Minnesota Multiphasic Personality Inventory profile of women with allergic rhinitis Psychosom Med 1993;55:533-40.

12. Addolorato G, Ancona C, Capristo E, Graziosetto R, Di Rienzo L, Maurizi M, Gasbarrini G. State and trait anxiety in women affected by allergic and vasomotor rhinitis. J Psychosom Res 1999;46:283-9.

13. van Os J, Jones PB. Neuroticism as a risk factor for schizophrenia. Psychol Med 2001;31:1129-34.

14. Bienvenu OJ, Nestadt G, Samuels JF, Costa PT, Howard WT, Eaton WW. Phobic, panic, and major depressive disorders and the five-factor model of personality. J Nerv Ment Dis 2001;189:154-61.

15. Bienvenu OJ, Samuels JF, Costa PT, Reti IM, Eaton WW, Nestadt G. Anxiety and depressive disorders and the five-factor model of personality: a higher- and lower-order personality trait investigation in a community sample. Depress Anxiety 2004;20:92-7.

16. Kessler RC, DuPont RL, Berglund P, Wittchen HU. Impairment in pure and comorbid generalized anxiety disorder and major depression at 12 months in two national surveys. Am J Psychiatry 1999;156:1915-23.

17. Kessler RC, Mickelson KD, Zhao S. Patterns and correlates of self-help group membership in the United States. Soc Policy 1997;27:27-46.
18. Wittchen HU. Reliability and validity studies of the WHO Composite International Diagnostic Interview (CIDI): a critical review. J Psychiatr Res 1994;28:57-84

19. Kessler RC, McGonagle KA, Zhao S, Nelson CB, Hughes M, Eshleman $\mathrm{S}$, Wittchen HU, Kendler KS. Lifetime and 12-month prevalence of DSM-III-R psychiatric disorders in the United States: results from the National Comorbidity Survey. Arch Gen Psychiatry 1994;51:8-19.

20. Kessler RC, Andrews G, Mrozek D, Ustun B, Wittchen HU. The World Health Organization Composite International Diagnostic Interview Short Form (CIDI-SF). Int J Methods Psychiatr Res 1998;7:171-85.

21. Lachman ME, Weaver SL. The Midlife Development Inventory (MIDI) Personality Scales: scale construction and scoring. Technical report. 1997.

22. Trapnell PD, Wiggins JS. Extension of the Interpersonal Adjective Scales to include the big five dimensions of personality. J Pers Soc Psychol 1990;59:781-90.

23. John OP. The "big five" factor taxonomy: dimensions of personality in the natural language and in questionnaires. In: Pervin LA, ed. Handbook of Personality Theory and Research. New York: Guilford; 1990:66-100.

24. Digman JM. Personality structure: emergence of the five-factor model. Ann Rev Psychol 41:417-40.

25. American Psychiatric Association. Diagnostic and Statistical Manual of Mental Disorders. Washington, DC: American Psychiatric Press; 1994.

26. Feldman PJ, Cohen S, Doyle WJ, Skoner DP, Gwaltney JM. The impact of personality on the reporting of unfounded symptoms and illness. J Pers Soc Psychol 1999;77:370-8.

27. Crystal-Peters J, Neslusan CA, Smith MW, Togias A. Health care costs of allergic rhinitis-associated conditions vary with allergy season. Ann Allergy Asthma Immunol 2002;89:457-62.

28. Leynaert B, Neukirch C, Liard R, Bousquet J, Neukirch F. Quality of life in allergic rhinitis and asthma.: a population-based study of young adults. Am J Respir Crit Care Med 2000;162:1391-6.

29. Kessler RC, Almeida DM, Berglund P, Stang P. Pollen and mold exposure impairs the work performance of employees with allergic rhinitis. Ann Allergy Asthma Immunol 2001;87:289-95.

30. Dantzer R, Bluthe RM, Laye S, Bret-Dibat JL, Parnet P, Kelley KW. Cytokines and sickness behavior. Ann N Y Acad Sci 1998;840:586-90.

31. Bell IR, Miller CS, Schwartz GE. An olfactory-limbic model of multiple chemical sensitivity syndrome: possible relationship to kindling and affective spectrum disorders. Biol Psychiatry 1992;32:218-42. 